In der Rubrik „Literatur kompakt" werden die wichtigsten Originalarbeiten aus der internationalen Fachliteratur referiert.

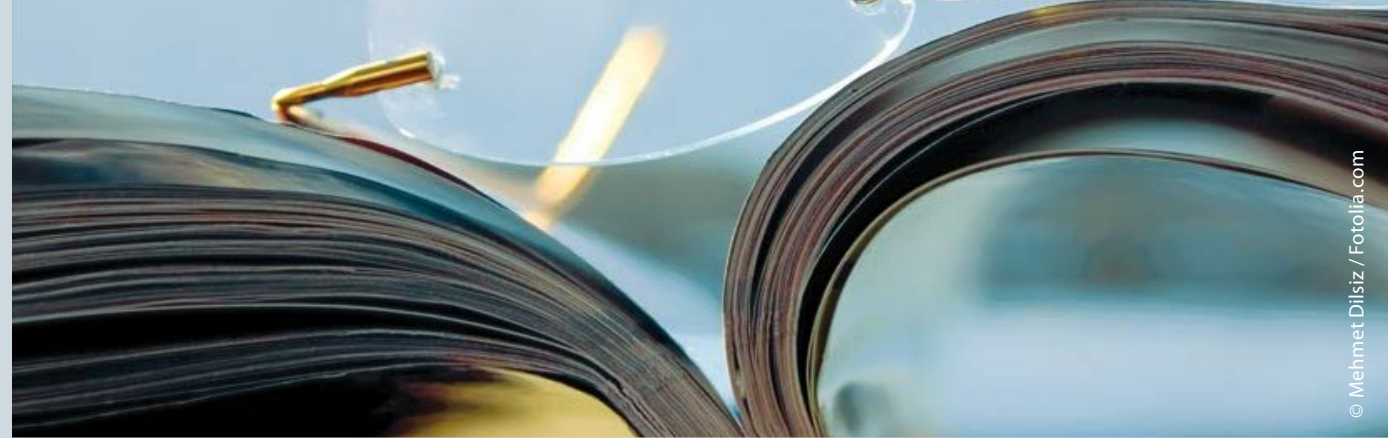

\section{Niedriger PSA-Wert kann ein schlechtes Vorzeichen sein}

\section{Üblicherweise sind niedrige PSA-Werte bei der Erstdiagnose eines Prostatakarzinoms mit einer besseren Prognose assoziiert. Bei Karzinomen vom Gleason-Grad 8-10 trifft das offenbar nicht zu. Werte $<4,0 \mathrm{ng} / \mathrm{ml}$ gehen hier mit einer aggressiveren Erkrankung einher als Werte von 4,0-9,9 ng/ml.}

$\mathrm{N}$ euentdeckte Prostatakarzinome mit einem Gleason-Score von $8-10$ und einem PSA $<4 \mathrm{ng} / \mathrm{ml}$ verhalten sich bezüglich ihrer klinischen Präsentation und der Überlebenschancen eher wie Karzinome mit einem PSA-Spiegel von 10,0-19,9 ng/ml. Das berichten Onkologen von der Universität in Chapel Hill im Journal Urologic Oncology. Die Ärzte um Aaron D. Falchook haben den Zusammenhang anhand von Patientendaten aus der National Cancer Data Base analysiert.

Berücksichtigt wurden 37.283 Männer, bei denen zwischen 2004 und 2007 schlecht- oder entdifferenzierte Prostatakarzinome entdeckt worden waren. $4.000(10,7 \%)$ hatten $\mathrm{zu}$ diesem Zeitpunkt einen PSA-Wert zwischen 0,1 und 3,9 ng/ml. Im Vergleich zu den $\mathrm{Pa}$ tienten mit PSA-Konzentrationen von 4,0-9,9 $\mathrm{ng} / \mathrm{ml}$ hatten sie ein höheres Risiko, dass sich der Tumor bereits über die Kapsel hinaus ausgebreitet (15,0\% vs. 9,7\%; p < 0,001) oder Metastasen in den Lymphknoten (3,8 \% vs. 2,1\%; $\mathrm{p}<0,001)$ oder Fernmetastasen gebildet hatte $(5,4 \%$ vs. $2,6 \%$; $p<0,001)$.

Wider Erwarten bestätigte sich ein ähnliches Bild bei Patienten, die einer Prostatektomie unterzogen wurden, jedoch nicht: In der Subgruppe von 8.896 Patienten mit Prostatektomie förderte das pathologische Staging bei PSA-Werten $<4 \mathrm{ng} / \mathrm{ml}$ mehr pT2- und pN0-Tumoren zutage als bei höheren PSA-Spiegeln.

Unabhängig von der First-Line-Behandlung - Operation oder Radiotherapie (bei $25 \%$ bzw. $46 \%$ angewendet) hatten Patienten mit einem PSA von 4,0-9,9 $\mathrm{ng} / \mathrm{ml}$ die besten Überlebenschancen. Bei PSA-Werten von 0,1-

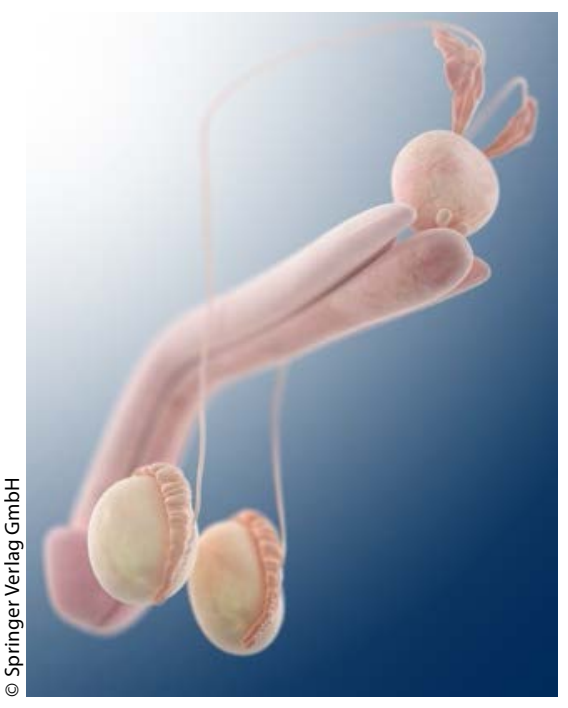

Ein niedriger PSA-Wert bei einem bösartigem Tumor in der Prostata kann die Überlebenschance des Patienten mindern.
$3,9 \mathrm{ng} / \mathrm{ml}$ waren sie dagegen ähnlich wie bei Werten von 10,0-19,9 ng/ml, und am schlechtesten waren die Aussichten bei Werten $\geq 20$ ng/ml. Die Sechs-JahresÜberlebensraten betrugen bei Prostatektomiepatienten mit PSA $<4,0 \mathrm{ng} / \mathrm{ml}$ $81,1 \%$ gegenüber $91,0 \%$ bei Werten von 4,0-9,9 ng/ml. Für Radiotherapiepatienten lauteten die entsprechenden Quoten 75,8 \% und $81,0 \%$. Die Unterschiede waren jeweils signifikant.

Fazit: Laut den US-Autoren entspricht ihre Beobachtung, dass ein niedriger PSA-Wert in dieser Patientengruppe mit einer höheren Wahrscheinlichkeit für eine fortgeschrittene Erkrankung und mit geringeren Überlebenschancen einhergeht, den Ergebnissen der beiden einzigen bisher zu diesem Thema publizierten Studien. Die niedrigen PSA-Spiegel seien in diesem Fall nicht Zeichen einer geringen Tumorlast, sondern vielmehr dem hohen Entdifferenzierungsgrad der Krebszellen geschuldet und damit das Resultat einer besonders aggressiven Erkrankung.

Dass bei den Prostatektomiepatienten in der pathologischen Untersuchung von Niedrig-PSA-Karzinomen keine erhöhte Rate an pT3/T4- oder pN1-Erkrankungen entdeckt wurde, könnte nach Ansicht von Falchook und Kollegen unter anderem an einer unterschiedlichen Patientenauswahl für die Prostatektomie gelegen haben.

Dr. Beate Schumacher

Falchook AD, Martin NE, Basak R et al. Stage at presentation and survival outcomes of patients with gleason 8-10 prostate cancer and low prostate-specific antigen. Urol Oncol. 2015, online 29. Oktober;doi:10.1016/j.urolonc.2015.09.014. 